\title{
Does the Use of Lycra-Hip-Orthoses Stabilize Gait and Increase Activity among Adults with Neurological Disabilities?
}

\author{
Grethe E Borchgrevink ${ }^{1 *}$, Tobias Gohil2,3, Per B Wik ${ }^{2}$, Kjerstin OE Selvig ${ }^{3}$ and Olav A Foss ${ }^{1,2}$ \\ ${ }^{1}$ Department of Orthopedic Surgery, St. Olav's University Hospital, Trondheim, Norway \\ ${ }^{2}$ Norwegian University of Science and Technology (NTNU), Trondheim, Norway \\ ${ }^{3}$ Trøndelag Orthopedic Workshop, Trondheim, Norway
}

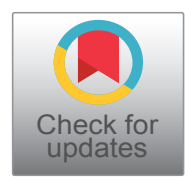

*Corresponding author: Grethe E Borchgrevink, Department of Orthopedic Surgery, St. Olav's University Hospital in Trondheim, Postbox 3250 Storgarden, 7006 Trondheim, Norway

\begin{abstract}
Background: The benefits of Lycra-garment-orthoses have not been convincing. We wanted to evaluate the effects of Lycra-hip-orthoses with several objective and subjective methods. The hypothesis was that the Lycra-hip-orthosis would improve gait, reduce lateral instability in the pelvis, reduce Trendelenburg gait and increase daily activity.

Methods: Ten patients with neurological disabilities with reduced stability of the pelvis were investigated at a $3 D$ gait-lab with and without Lycra-hip-orthoses at baseline and after a follow-up period of median 7.5 (range: 6-19) weeks using the Lycra-hip-orthosis. The patients also used an Accelerometer to measure activity and filled in questionnaires at baseline and endpoint.
\end{abstract}

Results: Apart from a reduction in anterior tilt of the pelvis, there were only minor changes for all the measured parameters.

Conclusion: We found no clinically relevant effects after wearing Lycra-hip-orthoses for a median follow-up time of 7.5 weeks.

\section{Keywords}

Neurological disease, Gait function, 3D gait-lab, Kinematics, Spatiotemporal data, Pelvic stability, Trendelenburg gait, Gait symmetry, Activity measurements

\section{Introduction}

Patients with neurological disabilities often have reduced stability of the pelvis while standing and walking, (positive Trendelenburg test) [1-3]. For these patients an orthototic device to stabilize their gait would be advantageous.
Traditionally, orthotic devices give mechanical constraints and are made of rigid materials such as thermoplastic and carbon fiber with integrated metal joints. Lycra-garment-orthoses allows more freedom of movement compared to those made of rigid materials.

The Lycra-garment-orthosis as a general concept was first introduced for children with cerebral palsy in the 1990s [4]. The Lycra-garment-orthoses are close fitting to give tactile stimulation and increase proprioception and body awareness [5]. Some sections are reinforced with stronger fabric to give strain/traction for individual biomedical needs [5], with the aim of correcting anatomical axes and rotational errors. The producers recommend the use of Lycra-orthoses to improve functional abilities [5]. There are many types of Lycra-garment-orthoses, but all are custom made for each patient and his/her instabilities.

The Lycra-hip-orthoses is marketed as an orthotic device to give a more anatomical position of the lower extremities, to give a better positioning of the hip muscles and in this way give a more stabilized gait [5]. The rigid hip-orthoses are abduction-orthoses made for use as time limited treatment-orthoses to prevent the hip from dislocating and are not suitable for use as orthotic devices.

Studies have tested the Lycra whole-body-orthoses or upper limb orthoses for children with cerebral palsy. The measurements most often have different observation assessment scales, such as the Gross Motor Func-

Citation: Borchgrevink GE, Gohil T, Wik PB, Selvig KOE, Foss OA (2020) Does the Use of Lycra-Hip-Orthoses Stabilize Gait and Increase Activity among Adults with Neurological Disabilities?. Int J Foot Ankle 4:047. doi.org/10.23937/2643-3885/1710047

Accepted: June 24, 2020: Published: June 26, 2020

Copyright: (c) 2020 Borchgrevink GE, et al. This is an open-access article distributed under the terms of the Creative Commons Attribution License, which permits unrestricted use, distribution, and reproduction in any medium, provided the original author and source are credited. 
Table 1: Patient-diagnoses.

\begin{tabular}{|l|l|l|}
\hline Diagnosis & Cerebral palsy: 2 & Born 1990 \\
\cline { 3 - 3 } & Multiple sclerosis: 4 & Born 1992 \\
\hline & & Diagnosed 1991 \\
\hline & Diagnosed 1996 \\
\hline & Charcot-Marie Tooth: 1 & Diagnosed 1995 \\
\hline & Sequela from cerebral hemorrhage: 1 & Diagnosed 2011 \\
\hline & Meningomyelocele: 1 & Insult 2015 \\
\hline & Slowly Progressive Spastic Palsy: 1 & Born 1962 \\
\hline
\end{tabular}

Table 2: Patient-characteristics at the time of recruitment.

\begin{tabular}{|l|l|l|}
\hline Sex & Men: 5 & Women: 5 \\
\hline Age (Range) & Median 58 years (26-76) & \\
\hline Height (Range) & Median 1.80 m (1.52-1.86) \\
\hline Weight (Range) & Median 86 kg (51-135) \\
\hline BMI (Range) & Median 29 (21-40) \\
\hline Need of crutches & 4 \\
\hline
\end{tabular}

tion Measure [6-8] and Pediatric Evaluation of Disability Inventory $[8,9]$. Some studies have used 3D gait analysis, one with spatiotemporal data such as stride length, cadence and gait speed [10] and others report data from kinematics [11,12]. In spite of great expectations for an orthosis without stiffness, review articles could not find evidence of improved function after using Lycra-orthoses [13-15]. They concluded that the effect is not evident and more studies are required.

We wanted to evaluate the effect of Lycra-hip-orthoses on adults with neurological disabilities and reduced pelvic stability (positive Trendelenburg test), to see if the Lycra-hip-orthoses would improve gait and pelvic stability and increase activity. We used objective and quantitative measurements from a 3D gait-laboratory and patients self-reported measures from questionnaires.

The hypothesis was that the Lycra-hip-orthosis would improve gait, reduce lateral instability of the pelvis, reduce Trendelenburg gait and increase daily activity.

\section{Material and Method}

This study is a prospective, explorative cohort study. It was approved by the Regional Committee for Medical and Health Research Ethics in central Norway (2017/2053). This ethics committee is appointed by the Norwegian Ministry for Education and Research.

\section{Patients}

Patients were recruited among referrals to Trøndelag Orthopedic Workshop AS (TOV) for clinical assessment and to consider the benefit of an orthopedic aid to improve their gait. Inclusion criteria were patients with neuromuscular disease who were clinically con- sidered to have positive Trendelenburg test, and with a minimum age of 18 years. The same orthopedic surgeon performed the clinical consultations. Exclusion criteria were patients without language, with mental deficiency and patients who needed an interpreter. The recruitment took place at TOV. Patients gave a written informed consent to participate in the study. Eleven patients were eligible, however, one patient did not manage to fulfill the whole study period and was excluded (Table 1 and Table 2).

\section{Experimental protocol}

This is a prospective, explorative cohort study. The ten included patients wore the Lycra-hip-orthosis for a minimum of 6 weeks, and they were instructed to wear the Lycra-hip-orthosis each day, seven hours a day, since this is what the producer describes are needed before expecting any effect.

\section{- 3D gait-lab}

The main measurement tool was testing in a 3D gait-lab with kinematics and spatiotemporal recordings data. The patients were investigated at the 3D gait-lab twice: At baseline and at the endpoint after a follow up period of at least 6 weeks.

The other measurement tools were:

- Daily activity measured with ActivePal ${ }^{\mathrm{TM}}$ accelerometer for one week at baseline and one week at endpoint.

- The EQ-5D-5L health questionnaire to assess the patients' subjective experience of health at baseline and at endpoint.

- Oxford scale for muscle strength measurement at baseline and at endpoint.

- At the endpoint we also inquired questions about the experience of wearing the Lycra-hip-orthosis.

\section{Instrumentations/Measurement tools}

3D-gait analysis: The gait analyses were performed in a 3D gait-lab using the Vicon 3D motion capture system and the Lower body Plug-in Gait model. Kinematic and spatiotemporal data were recorded. 


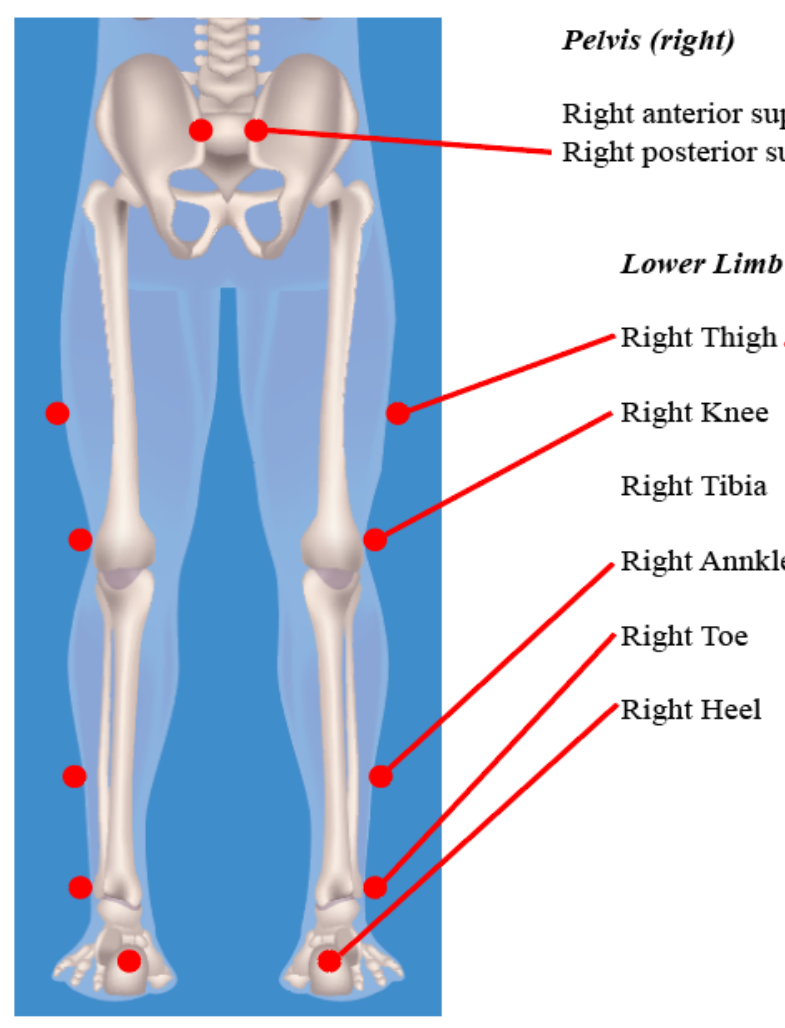

Posterior View

Figure 1: Markers localisations.
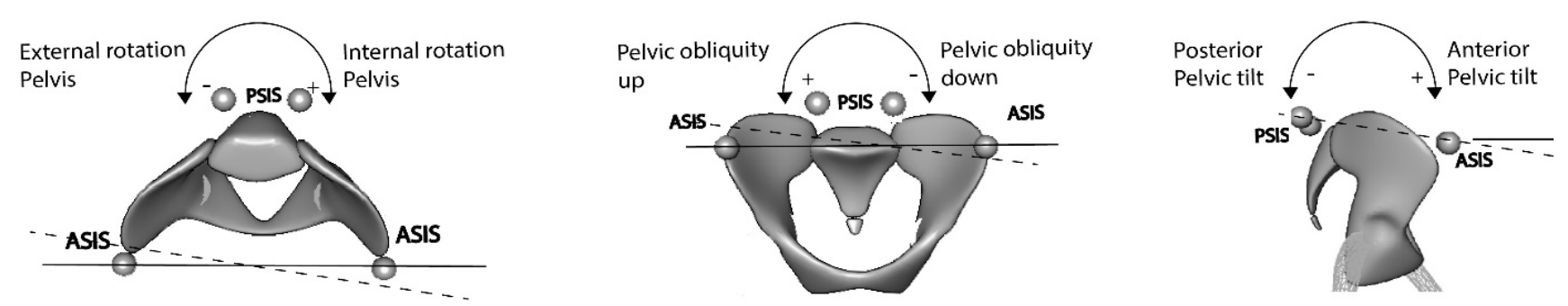

Figure 2: The 3D lab's coordinate system, rotation around three axes.

We used the Plug-In Gait-model (Vicon, Oxford UK), with 16 markers $14 \mathrm{~mm}$ in diameter, focusing on the lower extremity and 10 Vicon Vantage cameras. The Recording frequency was $200 \mathrm{~Hz}$. For the spatiotemporal data, we used three AMTI force-plates, (Model OR6-7 2000, $1000 \mathrm{~Hz}$; AMTI, Watertown, USA). The walkway at the gait-lab was eight meters. We attached the reflective markers directly onto the Lycra-hip-orthosis according to the conventional model described by Kadaba [16] (Figure 1).

The same engineer attached the reflective markers to all patients at all time-points. To consider any direct effect of Lycra-hip-orthosis, each session in the 3D gait-lab consisted of two different trial recordings, one wearing an ordinary sport-tights and one wearing the Lycra-hip-orthoses, and the markers were mounted in a similar position on the sport-tights. We chose sport-tights for the control in order to obtain a similar tight attachment for the reflective markers attached to the body. The patients walked at self-selected speed six rounds on the walkway with sport-tights and six rounds with the Lycra-hip-orthoses.

Kinematic data: From the kinematic data we chose to focus on the pelvis as our main aim was to assess possible effects from the Lycra-hip-orthoses on pelvic stability.

There were two anterior reflective markers at ASIS (Anterior Superior lliac Spine), and two posterior reflective markers at PSIS (Posterior Superior lliac Spine). These four reflective markers indicate the pelvic orientation and rotation in three planes (Figure 2).

The two anterior reflective markers at ASIS give a description of the frontal orientation (pelvic obliquity) relative to the plane of the floor (ASIS up, ASIS down), and the transverse orientation (pelvic rotation) relative to the line of forward walking progression (ASIS forward indicates pelvic internal rotation and ASIS backward indicates pelvic external rotation). The mid-point between the ASIS and the PSIS markers give a description of the sagittal orientation (pelvic tilt) relative to the floor (ASIS 
down and PSIS up indicate anterior tilt and ASIS up and PSIS down indicate posterior tilt).

The pelvic orientations and rotations were related to the 3 D-lab's global coordinate system which are presented as rotations about three axes in an orthogonal system. Two of the axes are relative to the laboratory floor ( $x$ - and $y$-axis): Rotation about the $x$-axis which is located in the walking direction describes pelvic obliquity in the frontal plane, and rotation about the $y$-axis which is perpendicular to the $\mathrm{x}$-axis, describes the pelvic tilt in sagittal plane. Rotation about the z-axis is a rotation in the transverse plane about the vertical axis and describes internal and external rotation (Figure 2).

3-D pelvic orientation and pelvic rotation data were collected at each test condition across all gait cycles.

The pelvic orientation was calculated as the grand mean value of degrees in the 3 planes (sagittal, frontal and transversal planes) and this describes the pelvic position during gait.

The pelvic stability was also based on the pelvic rotation data, and was calculated as the grand mean of the range (between max and min value of rotation in degrees) in the 3 planes (sagittal, frontal and transversal planes). The smaller the mean range value, the better the pelvic stability.

Spatiotemporal data: We chose to present single support (time-percentage of gait cycle), step length (meter), speed (meter pr. second) and cadence (steps pr. minute), as we wanted to assess any change in asymmetry in walking, and the possibility of improved walking progress.

If there is no limping the single support and step length in both feet should be equal, and by subtracting one foot from the other, the difference will be zero. We therefore made one single numeric value through subtracting one foot form the other. If the result differs from zero this means that the gait is disturbed. To make one single numeric value for cadence and speed, we added data from the right and left foot, and divided by two.

Activity: Activity was measured with ActivPal ${ }^{\mathrm{TM}}$ sensors. This is an accelerometer that classify daily activities into two conditions; sitting/lying down and standing/walking and these conditions were measured independently [17]. At baseline the patients, got the ActivePal ${ }^{\mathrm{TM}}$ attached to their right thigh, and were asked to leave it on for one week, and then return it to us. Covered by an adhesive tape, the ActivePal ${ }^{\mathrm{TM}}$ - sensor could be worn during showering. After the use of Lycra-hip-orthosis for at least six weeks (at endpoint), the patients again had the ActivePal ${ }^{\mathrm{TM}}$ attached to their right thigh and left it on for one week.

Questionnaires and Oxford scale: The EQ-5D-5L health questionnaire (to assess the patients' subjective experience of health), and the Oxford scale for manual assessment of muscle strength were completed by the same orthopedic surgeon together with the same orthopedic engineer at baseline and endpoint. At endpoint we also asked about the patients' experience wearing the Lycra-hip-orthosis. Information regarding the patients' actual use of the orthoses, including daily wear schedules were not recorded. Seven months after the end of the study period, we contacted the patients by telephone to ask them if the Lycra-hip-orthosis still was in use.

Statistics: The software package IBM SPSS Statistics for Windows version 25 (IBM Corp., Armonk, NY, USA) was used in the statistical analyses. Continuous outcome measurements were presented as box plots. The four sessions in the 3D gait-lab were named; Baseline Sport-tights, Baseline Lycra-hip-orthoses, Endpoint Sport-tights and Endpoint Lycra-hip-orthoses. Results from questionnaire and Oxford scale muscle strength were presented as means and range.

\section{Results}

Patients were recruited consecutively during a sixmonth period and collection of the data took place from 17.01.2018 to 01.03.2019. Ten patients completed the whole study period with complete data for all measurements.

The time between the baseline and endpoint investigations at the 3D gait-lab, was a median of 7.5 (range 6-19) weeks.

\section{D gait-lab}

Kinematic data pelvic orientation: There was a reduction in anterior tilt in the sagittal plane $\left(3.4^{\circ}\right)$, but there were only minor changes in orientation in the frontal $\left(-0.2^{\circ}\right)$ and transverse planes $\left(1.7^{\circ}\right)$ (Figure 3 ).

Kinematic data pelvic stability: There were only minor changes in stability with only minor changes in rotation about the $x$-axis $\left(0.3^{\circ}\right)$, and in rotation about the $y$-axis $\left(-0.3^{\circ}\right)$. For rotation about the $z$-axis there was a change of $-2.5^{\circ}$ (Figure 4 ).

Spatiotemporal data: There were only minor changes for single support $(-0.9 \%)$ and no changes in step length and thus no improvement of symmetry of gait. There was no change in speed and only minor changes in cadence ( 0.8 steps pr. $\mathrm{min}$ ) and thus no data to indicate improvement in walking progress (Figure 5).

\section{Activity}

There were no changes in daily activity between baseline and endpoint (Figure 6).

\section{Questionnaires and Oxford Scale}

We found a slight improvement of experienced health with the EQ-5D-5L questionnaire (Table 3). The Oxford Scale did show some improvements in muscle 


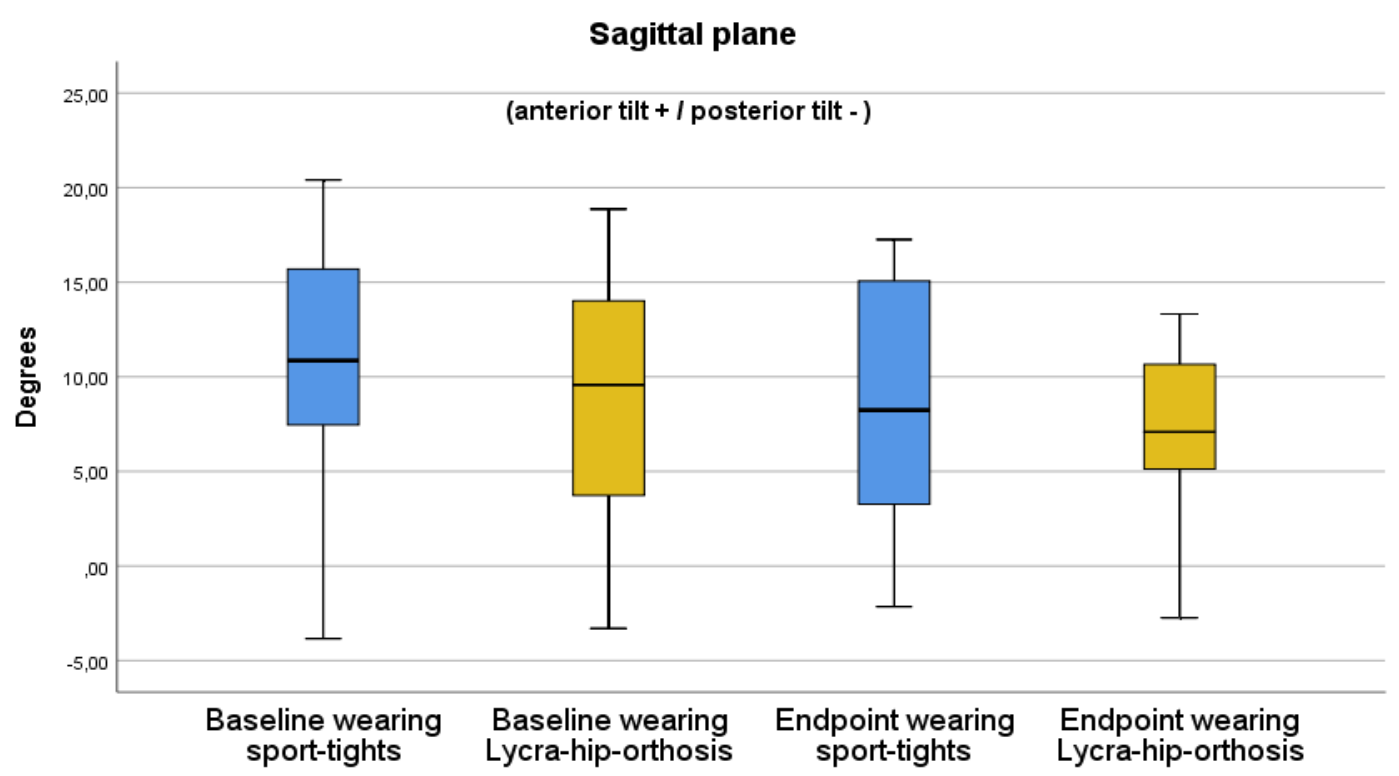

Frontal plane - Pelvic obliquity

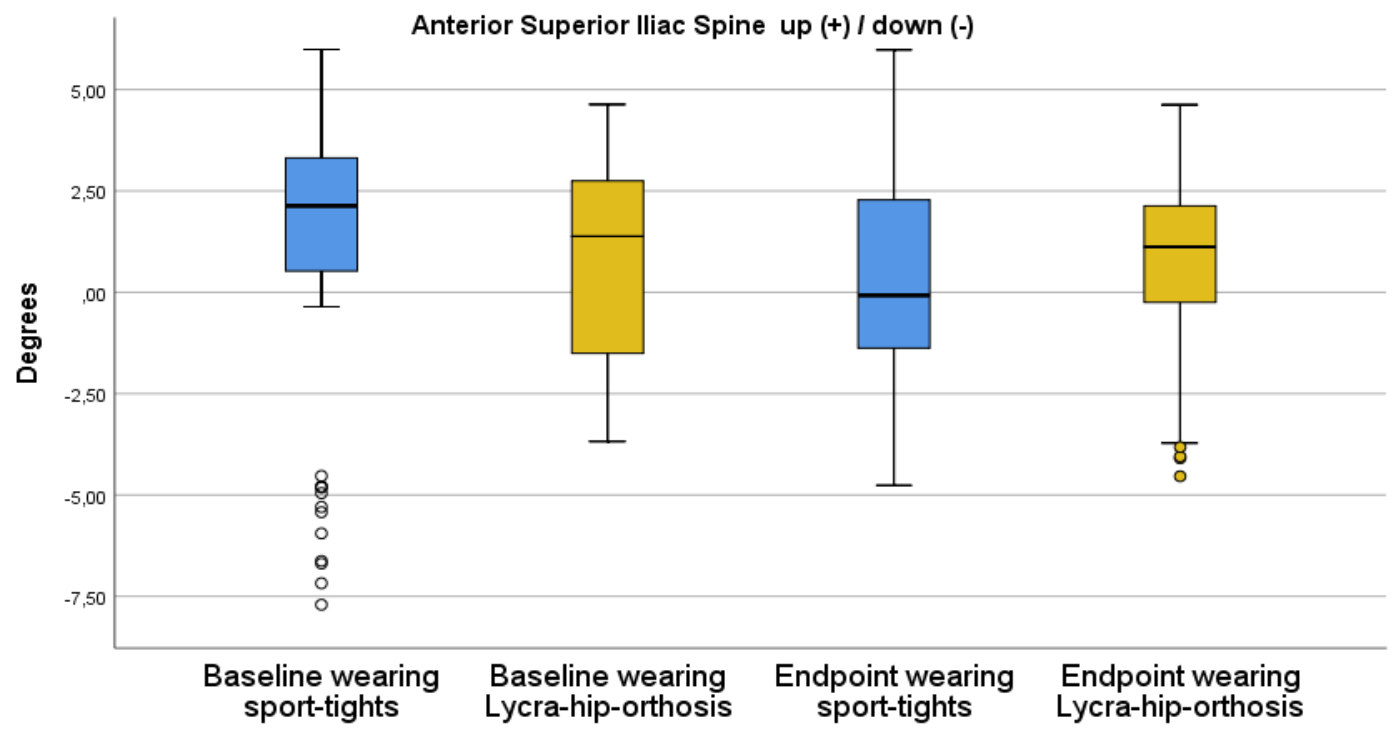

Transverse plane

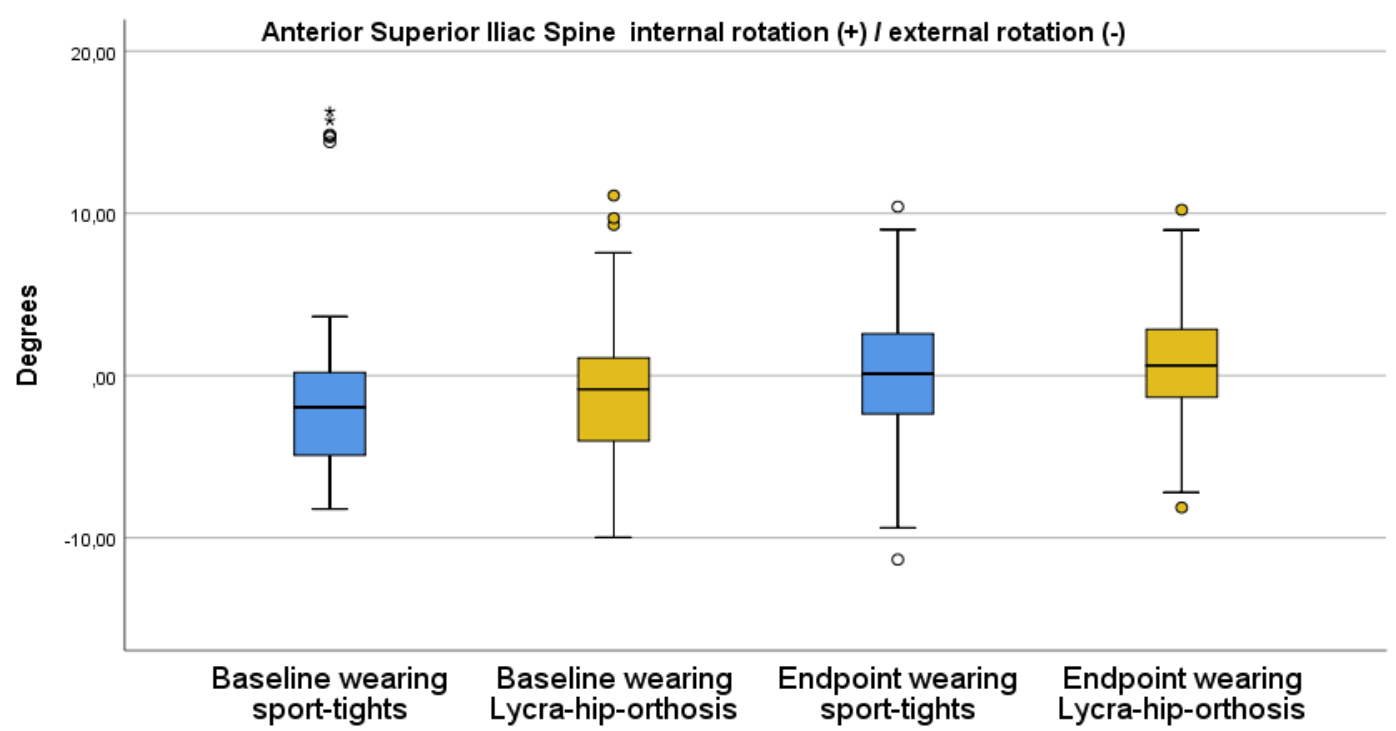

Figure 3: Kinematic data - Pelvic orientation. 

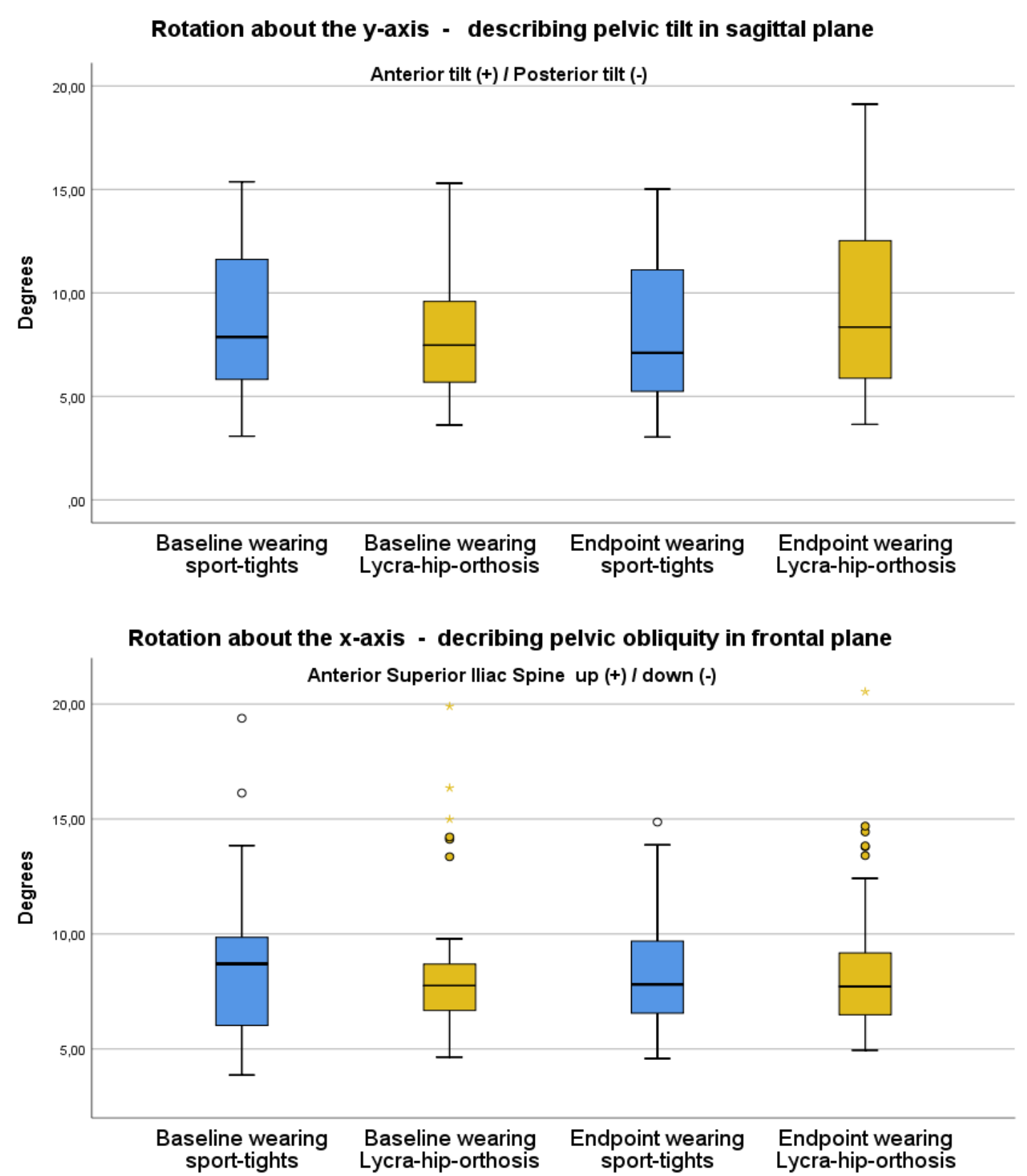

Rotation about the z-axis - decribing pelvic rotation in transverse plane

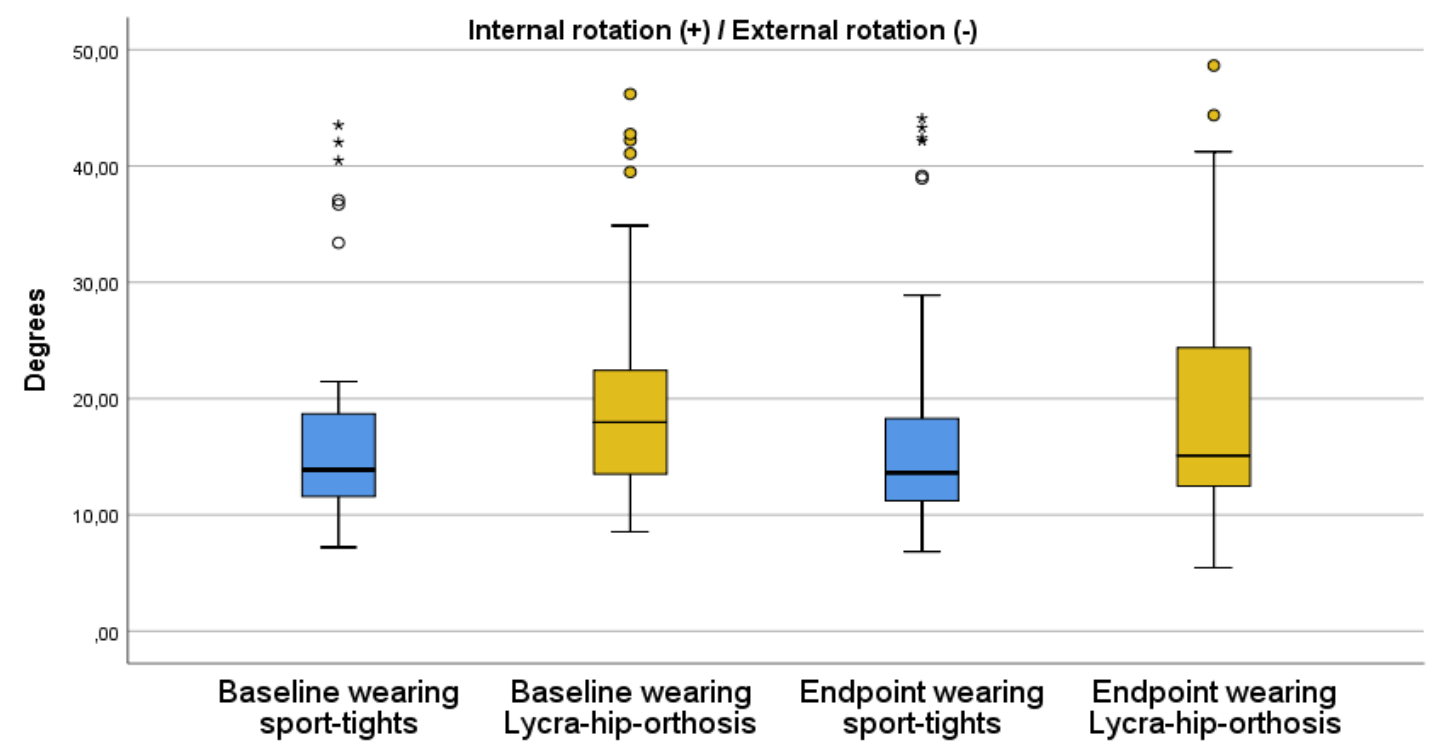

Figure 4: Kinematic data - Pelvic stability. 

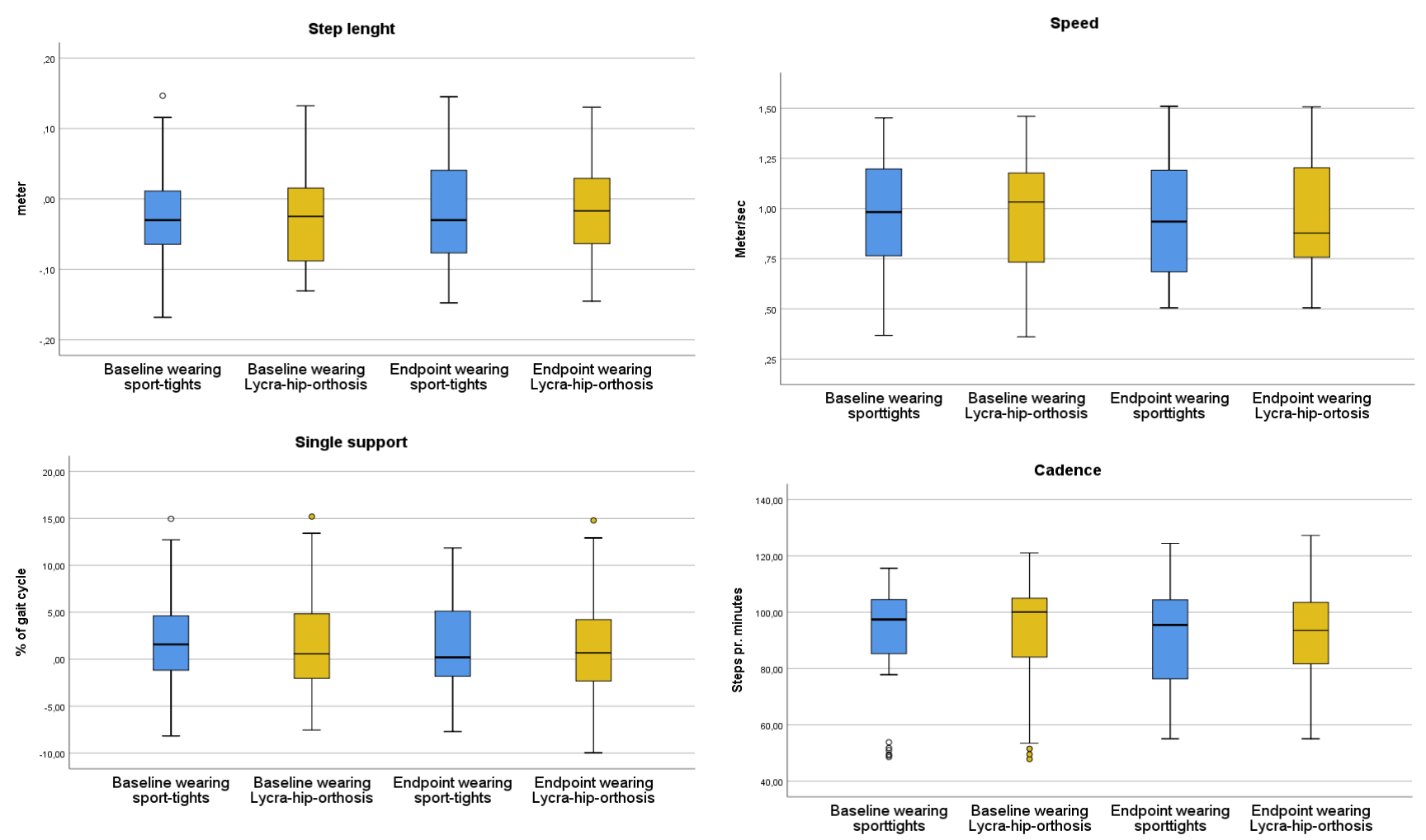

Figure 5: Spatiotemporal data.

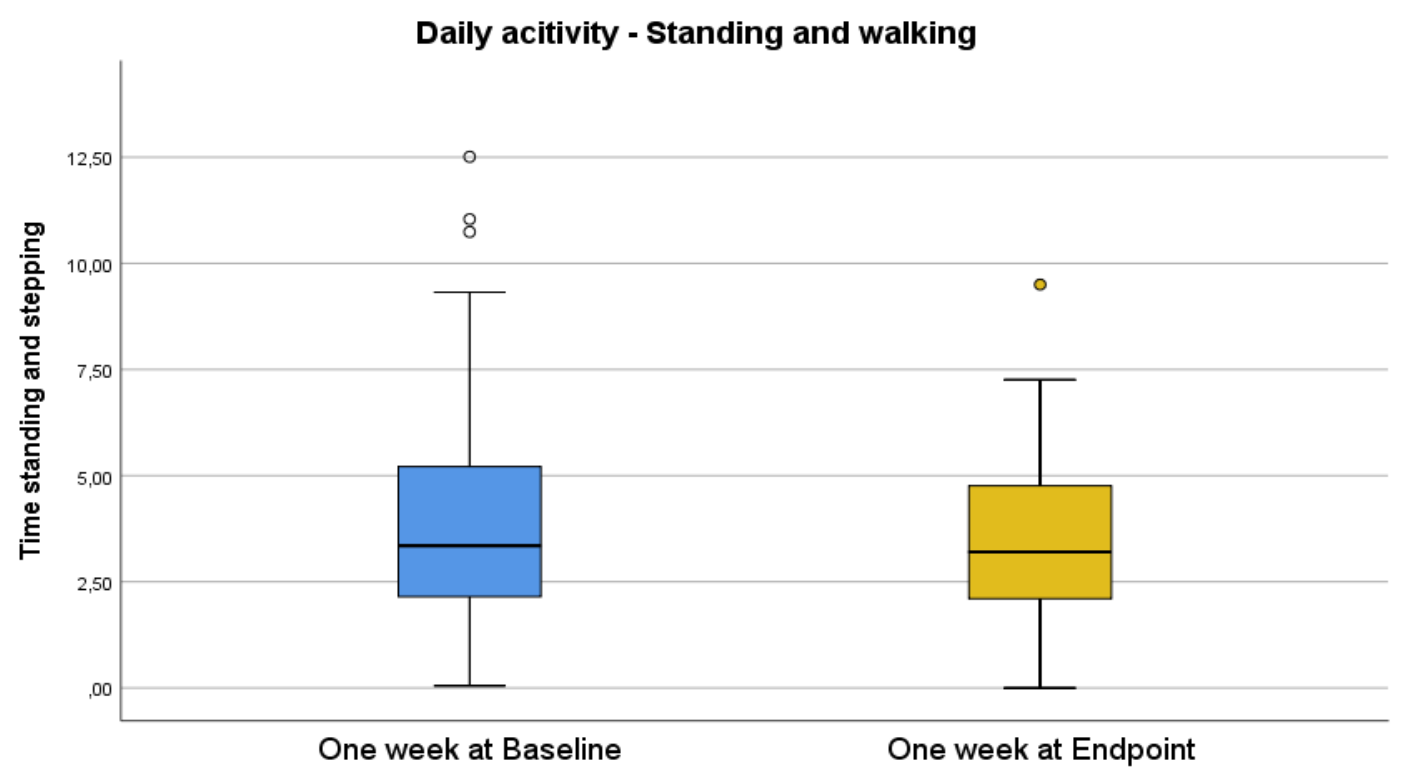

Figure 6: Activity.

strength (Table 4).

In the questionnaire to assess the patients' experiences with wearing the Lycra-hip-orthoses, the scores were better than the mean on the grading scale, but with a large range (Table 5). Six out of ten patients had chosen to continue wearing the Lycra-hip-orthoses after end of study as they felt that it stabilized their gait. One patient dropped the use of crutches while wearing the Lycra-hip-orthosis.

\section{Discussion}

We studied the effect of Lycra-hip-orthosis by using a broad range of objective and subjective measurements.

After the use of Lycra-hip-orthoses for a median time of 7.5 weeks, we found from the kinematics that the greatest change was a reduction $\left(3.4^{\circ}\right)$ in the anterior tilt of the pelvic orientation (Figure 3 ). A reduction of a pathological anterior tilt is favorable as it provides a basis for strengthened hip and trunk muscle power [18]. To assess whether the patients' anterior tilt in pelvis was pathological we had access to data for the pelvic orientation from a healthy population with age 
Table 3: EQ-5D-5L.

\begin{tabular}{|l|l|l|l|}
\hline & Mean at baseline & $\begin{array}{l}\text { Mean-difference between } \\
\text { baseline and endpoint }\end{array}$ & Range \\
\hline General health & 54.5 & +5 & 25 \\
\hline The specific questions are measured from 1-5, 1 , is worst, 5 is the best ability. & +0.5 & 0 \\
\hline Ability to walking about & 3.3 & 0.0 & 2 \\
\hline Problems washing or dressing myself & 4.4 & +0.1 & 5 \\
\hline Ability to do usual activities & 3.8 & +0.6 & 1 \\
\hline Pain or disorders & 3.2 & -0.2 & 1 \\
\hline Anxiety/depression & 4.5 & & 2 \\
\hline
\end{tabular}

General health is measured from $0-100,0$ is worse, 100 is the best health ever imagined.

Table 4: Oxford scale for muscle strength measurement.

\begin{tabular}{|l|l|l|l|}
\hline & Mean at baseline & $\begin{array}{l}\text { Mean-difference between } \\
\text { baseline and endpoint }\end{array}$ & Range \\
\hline Gluteus Medius muscle right & 2.7 & +1.0 & 4.5 \\
\hline Gluteus Maximus muscle right & 2.9 & +0.5 & 2.0 \\
\hline Gluteus Medius muscle left & 3.3 & +0.4 & 3.0 \\
\hline Gluteus Maximus muscle left & 3.1 & +0.4 & 6.0 \\
\hline
\end{tabular}

Grading $0-5,0$ is weak, 5 is strong.

Table 5: Experience of wearing the Lycra orthosis.

\begin{tabular}{|l|l|l|}
\hline & Mean at endpoint after the use of Lycra-hip-orthosis & Range \\
\hline Altogether how are your experience of using Lycra orthosis & 6.5 & 6 \\
\hline How was it to put on the orthosis & 6.1 & 8 \\
\hline How was it to take off the orthosis & 8.0 & 5 \\
\hline How did you feel the texture in the orthosis on your skin & 6.7 & 8 \\
\hline
\end{tabular}

Grading $1-10,1$ is difficult, 10 is no problem.

(40-60), from the same 3D gait-lab. The healthy population group were 10 persons ( 6 women and 4 men), with no gait problems or foot operation during the last years and with a mean height of $174 \mathrm{~cm}$ (range: $165-184 \mathrm{~cm}$ ) and weight of $75 \mathrm{~kg}$ (min $58 \mathrm{~kg}-\mathrm{max} 99 \mathrm{~kg}$ ). Our patients' anterior tilt before wearing the Lycra-hip-orthosis was $10.6^{\circ}$ (SD 6.1) versus the healthy population group's anterior tilt $9.0^{\circ}$ (SD 4.3), a difference of $1.6^{\circ}$.

Changes in rotation about the $x$-axis, describing pelvic obliquity in frontal plane (Figure 4) could have indicated a reduction in Trendelenburg gait, but was not supported by the results.

We could not find any published studies investigating the effect of Lycra-lower-extremity-orthoses on adults using 3D gait analysis. All the studies we found were in $\mathrm{CP}$-children.

Two studies presented kinematic data from the pelvis. Flanagan, et al. [11] found a reduction in the anterior pelvic tilt in five $\mathrm{CP}$ children after using an orthosis for 12 weeks. Rennie, et al. [12] found an improvement in proximal stability in eight $\mathrm{CP}$ children after wearing a "whole-body Lycra garment orthosis" for eight weeks, but the improvement was not statistically significant.
We found no improvement in gait symmetry or walking progress measured by spatiotemporal data (Figure 5). We could not find any fully comparable studies using spatiotemporal gait data to assess the effect of "under-wear Lycra/garment orthoses". Matthews, et al. [4] did not use 3D gait analysis and had limited quantitative data. They reported a statistically significant reduction of walking time after 18 weeks using a full-length "legging-Lycra-orthosis" in eight CP children. Abd El-Kafy, et al. [10] used stride length, cadence and speed to compare three different treatment interventions for children with spastic diplegic cerebral palsy. The interventions he used are not fully comparable to our study as all his intervention groups also underwent a physical therapy program. However, two of the intervention-groups in his study compared the effect of under-garment-orthoses; as one group used the garment-orthoses for 12 weeks, and the other group did not. After 12 weeks, there were no statistically significant differences for either cadence or speed, but there was a statistically significant larger stride length in the garment-orthoses-group.

Our results from the activity measurement did not show increased activity (Figure 6). We have not found any study using the ActivePal ${ }^{\mathrm{TM}}$ accelerometer to measure the effect of Lycra-hip-orthoses, but there are 
some review articles, which conclude that garment orthoses in general do not improve function [13-15].

Our hypothesis that the Lycra-hip-orthosis would improve gait, reduce lateral instability in the pelvis, reduce Trendelenburg gait and increase daily activity was not confirmed.

\section{Limitations}

The study was made without any advance strength calculation. This was because we could not find any study with relevant data from 3D gait analysis. The number of participants in the study may have been too small to show significant differences.

\section{Conclusion}

We found no clinically relevant effect from wearing Lycra-hip-orthosis for 7.5 weeks. The patients had no improvement in gait symmetry or walking forward progress, no increase in activity or evidence for a reduction in Trendelenburg gait. However, we did find a reduction in the patients' anterior tilt, which may be advantageous for better hip and trunk muscle power in the long run.

\section{Acknowledgements}

We would like to thank the users and staff at Trondelag Orthopedic Workshop in Trondheim. We also want to thank Siri Bjørgen Winther for her calculation of the ActivePal ${ }^{\mathrm{TM}}$ data, and Vilhjalmur Finsen for copyediting the manuscript into better English. The study was partly funded by Trondelag Orthopedic Workshop for which we are grateful.

\section{Conflict of Interest Statement}

We confirm that none of the authors have any conflict of interest.

\section{Author's Contribution}

Grethe E Borchgrevink (GEB) was the leader of the project.

Olav Foss was the statistician and a strong contributor to the manuscript; he also was responsible for the measurement with Activ ${ }^{\mathrm{TM}} \mathrm{Pal}$.

Kjerstin $\varnothing$ verlid Selvig was together with GEB the idea-maker for the study, and she made all the measurements for the Lycra-hip-orthosis.

Tobias Gohil are Head of the gait-lab, and together with Per-Bendik Wik were responsible for all the data collected at the 3D-gait lab.

\section{References}

1. Kiernan D, O'Sullivan R, Malone A, O'Brien T, Simms CK (2018) Pathological movements of the pelvis and trunk during gait in children with cerebral palsy: A cross-sectional study with 3-dimensional kinematics and lower lumbar spinal loading. Phys Ther 98: 86-94.
2. Metaxiotis D, Accles W, Siebel A, Doederlein L (2000) Hip deformities in walking patients with cerebral palsy. Gait Posture 11: 86-91.

3. Van lersel M, Mulley G (2004) What is a waddling gait? Disabil Rehabil 26: 678-682.

4. Matthews MJ, Watson M, Richardson B (2009) Effects of dynamic elastomeric fabric orthoses on children with cerebral palsy. Prosthet Orthot Int 33: 339-347.

5. https://www.bostonoandp.com/products/dynamic-movement-orthoses/

6. Knox V (2003) The use of lycra garments in children with cerebral palsy: A report of a descriptive clinical trial. $\mathrm{Br} \mathrm{J}$ Occup Therapy 66: 71-77.

7. Romeo DM, Specchia A, Sini F, Bompard S, Di Polito A, et al. (2018) Effects of lycra suits in children with cerebral palsy. Eur J Paediatr Neurol 22: 831-836.

8. Bailes AF, Greve K, Burch CK, Reder R, Lin L, et al. (2011) The effect of suit wear during an intensive therapy program in children with cerebral palsy. Pediatr Phys Ther 23: 136142.

9. Nicholsen JH, Morton RE, Attfield S, Rennie D (2001) Assessment of upper-limb function and movement in children with cerebral palsy wearing Lycra garment. Dev Med Child Neurol 43: 384-391.

10. Abd-El-Kafy EM (2014) The clinical impact of orthotic correction of lower limb rotational deformities in children with cerebral palsy: A randomized controlled trial. Clin Rehabil 28: 1004-1014.

11. Flanagan A, Krzak J, Peer M, Johnson P, Urban M (2009) Evaluation of short-term intensive orthotic garment use in children who have cerebral palsy. Pediatr Phys Ther 21: 201-204.

12. Rennie DJ, Attfield SF, Morton RE, Polak FJ, Nicholson J (2000) An evaluation of Lycra garments in lower limb using 3-D gait analysis and functional assessment (PEDI). Gait Posture 12: 1-6.

13. Wells H, Marques J, Wakely L (2018) Garment therapy does not improve function in children with cerebral palsy: A systematic review. Phys Occup Ther Pediatr 38: 395-416.

14. Almeida KM, Fonseca ST, Figueiredo PRP, Aquino AA, Mancini MC, et al. (2017) Effects of interventions with therapeutic suits (clothing) on impairments and functional limitations of children with cerebral palsy: A systematic review. Braz J Phys Ther 21: 307-320.

15. Martins E, Cordovil R, Oliveira R, Letras S, Lourenco S, et al. (2016) Efficacy of suit therapy on functioning in children and adolescents with cerebral palsy: A systematic review and meta-analysis. Dev Med Child Neurol 58: 348-360.

16. Kadaba MP, Ramakrishnan HK, Wootten ME (1990) Measurement of lower extremity kinematics during level walking. J Orthop Res 8: 383-392.

17. Evenson KR, Goto MM, Furberg RD (2015) Systematic review of the validity and reliability of consumer-wearable activity trackers. Int J Behav Nutr Phys Act 12: 159.

18. Tateuchi $\mathrm{H}$, Taniguchi $\mathrm{M}$, Mori N, Ichihashi N (2012) Balance of hip and trunk muscle activity is associated with increased anterior pelvic tilt during prone hip extension. $J$ Electromyogr Kinesiol 22: 391-397. 